\title{
First-order Intertwining Operators and Position-dependent Mass Schrödinger Equations in d Dimensions
}

\author{
C. Quesne \\ Physique Nucléaire Théorique et Physique Mathématique, Université Libre de Bruxelles, \\ Campus de la Plaine CP229, Boulevard du Triomphe, B-1050 Brussels, Belgium \\ E-mail: cquesne@ulb.ac.be
}

\begin{abstract}
The problem of $d$-dimensional Schrödinger equations with a position-dependent mass is analyzed in the framework of first-order intertwining operators. With the pair $\left(H, H_{1}\right)$ of intertwined Hamiltonians one can associate another pair of secondorder partial differential operators $\left(R, R_{1}\right)$, related to the same intertwining operator and such that $H$ (resp. $H_{1}$ ) commutes with $R$ (resp. $R_{1}$ ). This property is interpreted in superalgebraic terms in the context of supersymmetric quantum mechanics (SUSYQM). In the two-dimensional case, a solution to the resulting system of partial differential equations is obtained and used to build a physically-relevant model depicting a particle moving in a semi-infinite layer. Such a model is solved by employing either the commutativity of $H$ with some second-order partial differential operator $L$ and the resulting separability of the Schrödinger equation or that of $H$ and $R$ together with SUSYQM and shape-invariance techniques. The relation between both approaches is also studied.
\end{abstract}

PACS: 03.65.-w

Keywords: Schrödinger equation; Position-dependent mass; Intertwining Operator; Supersymmetry 


\section{INTRODUCTION}

The concept of position-dependent mass (PDM) is known to play an important role in the energy-density functional approach to the quantum many-body problem in the context of nonlocal terms of the accompanying potential. This formalism has been extensively used in nuclei [1], quantum liquids [2], ${ }^{3}$ He clusters [3], and metal clusters [4.

Another area wherein the PDM approximation provides a very useful tool is the study of electronic properties of many condensed-matter systems, such as semiconductors [5] and quantum dots [6]. In particular, recent progress in crystal-growth techniques (molecularbeam-epitaxy technique, for instance) for producing nonuniform semiconductor specimens, wherein the carrier effective mass depends on position, has considerably enhanced the interest in the theoretical description of semiconductor heterostructures.

Furthermore, PDM presence in quantum mechanical problems may reflect some other unconventional effects, such as a deformation of the canonical commutation relations or a curvature of the underlying space [7]. It has also recently been signalled in the rapidlygrowing field of PT-symmetric [8] (or, more generally, pseudo-Hermitian [9]) quantum mechanics as occurring in the Hermitian Hamiltonian equivalent to the PT-symmetric cubic anharmonic oscillator at lowest order of perturbation theory [10].

All these developments have stimulated the search for exact solutions of PDM quantum mechanical problems both in the nonrelativistic [7, 11, 12, 13, 14, 15, 16, 17, 18, 19, 20, 21, 22, 23, 24] and relativistic [25, 26] contexts since they may provide a conceptual understanding of some physical phenomena, as well as a testing ground for some approximation schemes. In the nonrelativistic case, which we are going to consider here, the generation of PDM and potential pairs leading to exactly solvable, quasi-exactly solvable or conditionally-exactly solvable Schrödinger equations has been achieved by extending some methods known in the constant-mass case, such as point canonical transformations [27], Lie algebraic methods [28], as well as supersymmetric quantum mechanical (SUSYQM) and shape-invariance techniques [29].

Most of these works have been devoted to one-dimensional systems. The aim of the present paper is to tackle the more difficult problem of generating exact solutions for $d$ dimensional PDM Schrödinger equations. For such a purpose, we will extend an approach that has proved very useful in one dimension, namely that of intertwining operators [19, related to SUSYQM methods (see also [7, 12, 13, 14, 15, 16, 18, 20, 21]). As a first step, 
we plan to consider here the case of first-order intertwining operators and their SUSYQM interpretation.

In Section 2, the general theory of $d$-dimensional PDM Hamiltonians admitting a firstorder intertwining operator is developed and a solution to the resulting system of partial differential equations is obtained in the two-dimensional case. From this solution, a new exactly-solvable PDM model in a semi-infinite layer is built in Section 3. In Subsections 3.1 and 3.2, the separability of the corresponding Schrödinger equation and the existence of an intertwining operator are shown to provide two alternative methods for getting the boundstate spectrum and wavefunctions. The relation between both approaches is then reviewed in Subsection 3.3. Section 4 contains some final remarks.

\section{GENERAL THEORY AND SUPERSYMMETRIC INTERPRETATION}

On using the von Roos general two-parameter form of the PDM kinetic energy operator [30], choosing units wherein $\hbar=2 m_{0}=1$ and summing over dummy indices, the $d$-dimensional PDM Schrödinger equation may be written as

$$
\left\{-\frac{1}{2}\left[M^{\alpha}(\boldsymbol{x}) \partial_{i} M^{\beta}(\boldsymbol{x}) \partial_{i} M^{\gamma}(\boldsymbol{x})+M^{\gamma}(\boldsymbol{x}) \partial_{i} M^{\beta}(\boldsymbol{x}) \partial_{i} M^{\alpha}(\boldsymbol{x})\right]+V(\boldsymbol{x})\right\} \psi(\boldsymbol{x})=E \psi(\boldsymbol{x}) .
$$

Here $\boldsymbol{x} \equiv\left(x_{1}, x_{2}, \ldots, x_{d}\right), \partial_{i} \equiv \partial / \partial x_{i}, i=1,2, \ldots, d, M(\boldsymbol{x})$ is the dimensionless form of the mass function $m(\boldsymbol{x})=m_{0} M(\boldsymbol{x}), V(\boldsymbol{x})$ is the potential and $\alpha, \beta, \gamma$ are the von Roos ambiguity parameters, constrained by the condition $\alpha+\beta+\gamma=-1$. The expression of the kinetic energy operator in (2.1) has the advantage of having an inbuilt Hermiticity

and of containing as special cases all the proposals made in the literature to cope with noncommutativity of the momentum and PDM operators.

As is well known (see, e.g., [7, 19]), we can get rid of the ambiguity parameters in the kinetic energy operator by transferring them to the effective potential energy of the variable mass system. Equation (2.1) therefore acquires the form

$$
H \psi(\boldsymbol{x}) \equiv\left(-\partial_{i} \frac{1}{M(\boldsymbol{x})} \partial_{i}+V_{\mathrm{eff}}(\boldsymbol{x})\right) \psi(\boldsymbol{x})=E \psi(\boldsymbol{x})
$$

where

$$
V_{\text {eff }}(\boldsymbol{x})=V(\boldsymbol{x})+\frac{1}{2}(\beta+1) \frac{\Delta M}{M^{2}}-[\alpha(\alpha+\beta+1)+\beta+1] \frac{\left(\partial_{i} M\right)\left(\partial_{i} M\right)}{M^{3}}
$$


and $\Delta \equiv \partial_{i} \partial_{i}$ denotes the $d$-dimensional Laplacian operator.

In the following, we shall be interested in the bound-state energy spectrum $E_{n}, n=0$, $1,2, \ldots$, of $H$ and in the corresponding wavefunctions $\psi_{n}(\boldsymbol{x}), n=0,1,2, \ldots$ To be physically acceptable, the latter should satisfy two conditions [20]:

(i) As in conventional quantum mechanics with a constant mass, they should be square integrable over the domain $D$ on which $M(\boldsymbol{x})$ and $V(\boldsymbol{x})$ are defined, i.e.,

$$
\int_{D} d \boldsymbol{x}\left|\psi_{n}(\boldsymbol{x})\right|^{2}<\infty
$$

(ii) To ensure that $H$ be Hermitian in the Hilbert space to which the $\psi_{n}(\boldsymbol{x})$ 's belong, they should be such that

$$
\frac{\left|\psi_{n}(\boldsymbol{x})\right|^{2}}{\sqrt{M(\boldsymbol{x})}} \rightarrow 0 \quad \text { on the boundary of } D
$$

This extra condition may have relevant effects whenever $M(\boldsymbol{x})$ vanishes at some boundary point.

Let us consider the intertwining relationship

$$
\eta H=H_{1} \eta
$$

where $H_{1}$ has the same kinetic energy term as $H$ and an associated effective potential $V_{1, \text { eff }}(\boldsymbol{x})$. Choosing a first-order intertwining operator

$$
\eta=A^{(i)}(\boldsymbol{x}) \partial_{i}+B(\boldsymbol{x})
$$

we are led to the restrictions

$$
\begin{aligned}
& \partial_{i} A^{(j)}+\partial_{j} A^{(i)}=-\delta_{i, j} A^{(k)} \frac{\partial_{k} M}{M}, \\
& A^{(i)}\left(V_{\mathrm{eff}}-V_{1, \mathrm{eff}}\right)=-A^{(j)}\left(\frac{\partial_{i} \partial_{j} M}{M^{2}}-2 \frac{\left(\partial_{i} M\right)\left(\partial_{j} M\right)}{M^{3}}\right)-\frac{1}{M} \Delta A^{(i)} \\
& +\left(\partial_{j} A^{(i)}\right) \frac{\partial_{j} M}{M^{2}}-\frac{2}{M} \partial_{i} B \\
& A^{(i)} \partial_{i} V_{\mathrm{eff}}=-B\left(V_{\mathrm{eff}}-V_{1, \mathrm{eff}}\right)-\frac{1}{M} \Delta B+\left(\partial_{i} B\right) \frac{\partial_{i} M}{M^{2}} .
\end{aligned}
$$

From the intertwining relation (2.6) and the Hermiticity of $H$ and $H_{1}$, it follows that

$$
H \eta^{\dagger}=\eta^{\dagger} H_{1}
$$


showing that

$$
R \equiv \eta^{\dagger} \eta, \quad R_{1} \equiv \eta \eta^{\dagger}
$$

are such that

$$
[H, R]=0, \quad\left[H_{1}, R_{1}\right]=0 .
$$

Hence $R$ is an integral of motion for the quantum mechanical problem described by $H$ (provided it is independent of the latter). A similar situation occurs for the pair $\left(H_{1}, R_{1}\right)$. Inserting $\eta$ from (2.7) and $\eta^{\dagger}=-A^{(i)} \partial_{i}-\left(\partial_{i} A^{(i)}\right)+B$ in (2.12), we get

$$
\begin{aligned}
R & =-A^{(i)} A^{(j)} \partial_{i j}^{2}-\left[A^{(i)}\left(\partial_{j} A^{(j)}\right)+A^{(j)}\left(\partial_{j} A^{(i)}\right)\right] \partial_{i}-A^{(i)}\left(\partial_{i} B\right)-B\left(\partial_{i} A^{(i)}\right)+B^{2}, \\
R_{1} & =R-A^{(i)}\left[\left(\partial_{i j}^{2} A^{(j)}\right)-2\left(\partial_{i} B\right)\right] .
\end{aligned}
$$

The results obtained so far are amenable to a superalgebraic interpretation. Since

$$
\eta R=R_{1} \eta, \quad R \eta^{\dagger}=\eta^{\dagger} R_{1}
$$

we may indeed introduce some matrix operators

$$
\mathcal{H}=\left(\begin{array}{cc}
H & 0 \\
0 & H_{1}
\end{array}\right), \quad \mathcal{R}=\left(\begin{array}{cc}
R & 0 \\
0 & R_{1}
\end{array}\right), \quad \mathcal{Q}^{+}=\left(\begin{array}{cc}
0 & 0 \\
\eta & 0
\end{array}\right), \quad \mathcal{Q}^{-}=\left(\begin{array}{cc}
0 & \eta^{\dagger} \\
0 & 0
\end{array}\right)
$$

satisfying the defining relations of the $\mathrm{gl}(1 / 1)$ superalgebra [31], i.e.,

$$
\left\{\mathcal{Q}^{+}, \mathcal{Q}^{-}\right\}=0, \quad\left\{\mathcal{Q}^{ \pm}, \mathcal{Q}^{ \pm}\right\}=0, \quad\left[\mathcal{R}, \mathcal{Q}^{ \pm}\right]=\left[\mathcal{H}, \mathcal{Q}^{ \pm}\right]=[\mathcal{H}, \mathcal{R}]=0
$$

In such a context, $\mathcal{R}, \mathcal{Q}^{+}$and $\mathcal{Q}^{-}$realize the usual SUSYQM sl(1/1) superalgebra [29], while $\mathcal{H}$ turns out to be the Casimir operator of $\mathrm{gl}(1 / 1)$.

In the one-dimensional case, it has been shown elsewhere [19] that the solution of Eqs. (2.8) - (2.10) can be written as

$$
A(x)=M^{-1 / 2}, \quad V_{\mathrm{eff}}(x)=-(A B)^{\prime}+B^{2}+\lambda, \quad V_{1, \mathrm{eff}}(x)=V_{\mathrm{eff}}-A\left(A^{\prime \prime}-2 B^{\prime}\right),
$$

where $\lambda$ is some integration constant. Note that from (2.18) onwards, we denote by a prime the derivative of any function depending on a single variable. On combining now (2.18) with (2.14), we arrive at the trivial outcome

$$
R=H-\lambda, \quad R_{1}=H_{1}-\lambda .
$$


To get significant results in (2.12) - (2.17), we must therefore be at least in two dimensions. In such a case, the existence of the integral of motion $R$ (resp. $R_{1}$ ) means that $H$ (resp. $\left.H_{1}\right)$ is integrable. The first constraint (2.8) can now be written as

$$
\partial_{x} A^{(1)}=-\frac{1}{2 M}\left(A^{(1)} \partial_{x} M+A^{(2)} \partial_{y} M\right), \quad \partial_{y} A^{(1)}=-\partial_{x} A^{(2)}, \quad \partial_{x} A^{(1)}=\partial_{y} A^{(2)},
$$

where we henceforth denote $x_{1}, x_{2}, \partial_{1}, \partial_{2}$ by $x, y, \partial_{x}, \partial_{y}$, respectively. Conditions (2.20) imply that

$$
\Delta A^{(1)}=\Delta A^{(2)}=0,
$$

thus leading to a small simplification in the second constraint (2.9).

Let us find the general solution to Eq. (2.20) in the special case where the PDM depends on a single variable, e.g.,

$$
M=M(x) .
$$

The first constraint in (2.20) then amounts to

$$
\frac{\partial_{x} A^{(1)}}{A^{(1)}}=-\frac{M^{\prime}}{2 M}
$$

so that

$$
A^{(1)}=\frac{f(y)}{\sqrt{M}},
$$

where $f(y)$ is a so far undetermined function of $y$. Plugging (2.24) in the second constraint of (2.20), we find

$$
A^{(2)}=-f^{\prime}(y) \int^{x} \frac{d x^{\prime}}{\sqrt{M\left(x^{\prime}\right)}}+g(y)
$$

in terms of another undetermined function $g(y)$. It remains to solve the third constraint in (2.20), which can be rewritten as

$$
f(y)\left(\frac{1}{\sqrt{M}}\right)^{\prime}=-f^{\prime \prime}(y) \int^{x} \frac{d x^{\prime}}{\sqrt{M\left(x^{\prime}\right)}}+g^{\prime}(y) .
$$

On deriving both sides of this relation with respect to $x$, we arrive at the conclusion that

$$
\frac{f^{\prime \prime}(y)}{f(y)}=-\frac{(1 / \sqrt{M(x)})^{\prime \prime}}{1 / \sqrt{M(x)}}=C,
$$

where $C$ is some constant. Let us now distinguish between the three subcases $C<0, C=0$ and $C>0$. 
Whenever $C=-q^{2}<0$ (where we may assume $q>0$ ), we find from (2.27)

$$
f(y)=a \sin q y+b \cos q y, \quad \frac{1}{\sqrt{M}}=c \sinh q x+d \cosh q x,
$$

where $a, b, c, d$ are four integration constants. Inserting these relations in (2.26) shows that $g(y)$ must reduce to some constant $g$. We conclude that in the first subcase, the general solution of (2.20), corresponding to the choice (2.22), is given by

$$
\begin{aligned}
M(x) & =\frac{1}{(c \sinh q x+d \cosh q x)^{2}}, \\
A^{(1)}(x, y) & =(a \sin q y+b \cos q y)(c \sinh q x+d \cosh q x), \\
A^{(2)}(x, y) & =(-a \cos q y+b \sin q y)(c \cosh q x+d \sinh q x)+g .
\end{aligned}
$$

By proceeding in a similar way for $C=0$ and $C=q^{2}$ (with $q>0$ ), we respectively obtain

$$
\begin{aligned}
M(x) & =\frac{1}{(c x+d)^{2}}, \\
A^{(1)}(x, y) & =(a y+b)(c x+d), \\
A^{(2)}(x, y) & =-\frac{1}{2} a c\left(x^{2}-y^{2}\right)-a d x+b c y+g,
\end{aligned}
$$

and

$$
\begin{aligned}
M(x) & =\frac{1}{(c \sin q x+d \cos q x)^{2}}, \\
A^{(1)}(x, y) & =(a \sinh q y+b \cosh q y)(c \sin q x+d \cos q x), \\
A^{(2)}(x, y) & =(a \cosh q y+b \sinh q y)(c \cos q x-d \sin q x)+g,
\end{aligned}
$$

in terms of five arbitrary constants $a, b, c, d, g$.

Considering next Eq. (2.9) in the case where $M$ is given by (2.22), we get

$$
\begin{aligned}
& A^{(1)}\left(V_{\mathrm{eff}}-V_{1, \mathrm{eff}}\right)=-\frac{2 C}{M} A^{(1)}-\frac{2}{M} \partial_{x} B, \\
& A^{(2)}\left(V_{\mathrm{eff}}-V_{1, \mathrm{eff}}\right)=-\left(\frac{1}{M}\right)^{\prime} \partial_{x} A^{(2)}-\frac{2}{M} \partial_{y} B,
\end{aligned}
$$

where use has been made of Eq. (2.27).

To go forward in the solution of such relations, it is appropriate to make some specific choice for $C$ and for the five integration constants $a, b, c, d, g$. Here we consider the first 
subcase referred to hereabove and therefore assume $C=-q^{2}<0$. Some comments on the two remaining subcases will be postponed until Section 4 . On taking $a=d=1$ and $b=c=g=0$ in (2.29), we arrive at

$$
M(x)=\operatorname{sech}^{2} q x, \quad A^{(1)}(x, y)=\cosh q x \sin q y, \quad A^{(2)}(x, y)=-\sinh q x \cos q y .
$$

Note that with this choice, the mass is well defined on the whole real line (which would not happen if $c$ did not vanish). Furthermore, $q$ may be treated as a deformation parameter so that when $q \rightarrow 0$, we get back the constant-mass case $M(x) \rightarrow 1$.

The two conditions in (2.32) can now be rewritten as

$$
V_{\text {eff }}-V_{1, \text { eff }}=2 q^{2} \cosh ^{2} q x-2 \cosh q x \csc q y \partial_{x} B
$$

and

$$
V_{\mathrm{eff}}-V_{1, \mathrm{eff}}=-2 q^{2} \cosh ^{2} q x+2 \cosh q x \operatorname{coth} q x \sec q y \partial_{y} B,
$$

respectively. On equating their right-hand sides and setting $B(x, y)=\sin q y \beta(x, y)$, we get a separable first-order partial differential equation for $\beta(x, y)$. Its general solution leads to

$$
B(x, y)=(q \sinh q x+F \operatorname{csch} q x) \sin q y+G,
$$

where $F$ and $G$ denote some integration constants. From (2.34) and (2.36), we then obtain

$$
V_{\text {eff }}-V_{1, \mathrm{eff}}=2 q F \operatorname{coth}^{2} q x .
$$

Finally it remains to solve Eq. (2.10). On taking (2.33), (2.36) and (2.37) into account, it can be easily transformed into the partial differential equation

$$
\begin{aligned}
& \operatorname{coth} q x \partial_{x} V_{\text {eff }}-\cot q y \partial_{y} V_{\text {eff }} \\
& \quad=-2 q\left[q^{2} \cosh ^{2} q x+F(q+F) \operatorname{csch}^{2} q x \operatorname{coth}^{2} q x+F G \operatorname{csch} q x \operatorname{coth}^{2} q x \csc q y\right] .
\end{aligned}
$$

We observe that such an equation is separable provided we choose a vanishing integration constant $G$. In this case, we obtain

$$
\begin{gathered}
V_{\text {eff }}(x, y)=-q^{2} \cosh ^{2} q x+F(q+F) \operatorname{csch}^{2} q x+J \ln (\cosh q x \sec q y)+K, \\
V_{1, \text { eff }}(x, y)=-q^{2} \cosh ^{2} q x+F(-q+F) \operatorname{csch}^{2} q x+J \ln (\cosh q x \sec q y)+K-2 q F,
\end{gathered}
$$

in terms of two additional integration constants $J$ and $K$. 
We conclude that Eqs. (2.33), (2.36) (with $G=0$ ), (2.39) and (2.40) provide us with a particular solution to the system of partial differential equations (2.8) $-(2.10)$ in the $d=2$ case. In order that this solution be viable and useful in the context of PDM Schrödinger equations with a bound-state spectrum, we have to impose some additional restrictions. This will be the purpose of Section 3, where we shall proceed to construct and to solve an interesting two-dimensional PDM model.

\section{A NEW EXACTLY-SOLVABLE PDM MODEL IN A SEMI-INFINITE LAYER}

Let us consider a particle moving in a semi-infinite layer of width $\pi / q$, parallel to the $x$-axis and with impenetrable barriers at the boundaries. This means that the variables $x, y$ vary in the domain

$$
D: \quad 0<x<\infty, \quad-\frac{\pi}{2 q}<y<\frac{\pi}{2 q},
$$

and that the wavefunctions have to satisfy the conditions

$$
\psi(0, y)=0, \quad \psi\left(x, \pm \frac{\pi}{2 q}\right)=0 .
$$

The motion of the particle is assumed to be described by the Hamiltonian

$$
H^{(k)}=-\partial_{x} \cosh ^{2} q x \partial_{x}-\cosh ^{2} q x \partial_{y}^{2}-q^{2} \cosh ^{2} q x+q^{2} k(k-1) \operatorname{csch}^{2} q x+q^{2} v_{0}
$$

corresponding to the mass and the effective potential given in (2.33) and (2.39), where in the latter equation we have set $J=0$ to get an acceptable potential, as well as $F=-q k$ (with $k>0$ ) and $K=q^{2} v_{0}$ (with $v_{0}$ arbitrary).

From the results of Section 2, it follows that $H^{(k)}$ and

$$
H_{1}^{(k)}=-\partial_{x} \cosh ^{2} q x \partial_{x}-\cosh ^{2} q x \partial_{y}^{2}-q^{2} \cosh ^{2} q x+q^{2} k(k+1) \operatorname{csch}^{2} q x+q^{2}\left(v_{0}+2 k\right)
$$

admit the intertwining operator

$$
\eta^{(k)}=\sin q y\left(\cosh q x \partial_{x}+q \sinh q x-q k \operatorname{csch} q x\right)-\sinh q x \cos q y \partial_{y}
$$

and that their respective constants of motion read

$$
\begin{aligned}
R^{(k)}= & -\cosh ^{2} q x \sin ^{2} q y \partial_{x}^{2}+2 \sinh q x \cosh q x \sin q y \cos q y \partial_{x y}^{2}-\sinh ^{2} q x \cos ^{2} q y \partial_{y}^{2} \\
& +q \sinh q x \cosh q x\left(1-4 \sin ^{2} q y\right) \partial_{x}+q \sin q y \cos q y\left(1+4 \sinh ^{2} q x\right) \partial_{y} \\
& +q^{2}\left(\sinh ^{2} q x-\sin ^{2} q y-3 \sinh ^{2} q x \sin ^{2} q y\right)-q^{2} k\left(1+\operatorname{csch}^{2} q x \sin ^{2} q y\right) \\
& +q^{2} k^{2} \operatorname{csch}^{2} q x \sin ^{2} q y
\end{aligned}
$$


and

$$
R_{1}^{(k)}=R^{(k)}+2 q^{2} k\left(1+\operatorname{csch}^{2} q x \sin ^{2} q y\right)
$$

The bound-state energy spectrum and wavefunctions of $H^{(k)}$ can be determined in two alternative ways: either by directly solving the corresponding Schrödinger equation, which is separable in the variables $x, y$, or by taking advantage of the intertwining operator $\eta^{(k)}$. We plan to successively review both approaches. Then, in a third step, we will determine the relation between the two resulting sets of wavefunctions.

\subsection{Separation of Variables in the PDM Schrödinger Equation}

Let us consider the Schrödinger equation (2.2) for $H^{(k)}$ with corresponding energy eigenvalues and wavefunctions denoted by $E^{(k)}$ and $\psi^{(k)}(x, y)$, respectively. From (3.3)), it is clear that the operator

$$
L \equiv-\partial_{y}^{2}
$$

commutes with $H^{(k)}$, so that both operators are simultaneously diagonalizable or, in other words, Eq. (2.2) is separable in the variables $x, y$.

The dependence of the wavefunctions on $y$ is determined by the solution of the eigenvalue problem for $L$ on the interval $-\pi /(2 q)<y<\pi /(2 q)$ with boundary conditions coming from the second relation in (3.2). One easily finds

$$
L \chi_{l}(y)=(l+1)^{2} q^{2} \chi_{l}(q), \quad l=0,1,2, \ldots,
$$

where the (normalized) eigenfunctions are given by

$$
\chi_{l}(y)=\left\{\begin{array}{ll}
\sqrt{\frac{2 q}{\pi}} \cos [(l+1) q y] & \text { for } l=0,2,4, \ldots \\
\sqrt{\frac{2 q}{\pi}} \sin [(l+1) q y] & \text { for } l=1,3,5, \ldots
\end{array} .\right.
$$

The simultaneous eigenfunctions of $H^{(k)}$ and $L$ can therefore be written as

$$
\psi_{n, l}^{(k)}(x, y)=\phi_{n, l}^{(k)}(x) \chi_{l}(y),
$$

where $\phi_{n, l}^{(k)}(x)$ satisfies the ordinary differential equation

$$
\begin{aligned}
H_{l}^{(k)} \phi_{n, l}^{(k)}(x) & \\
\equiv & \left(-\cosh ^{2} q x \frac{d^{2}}{d x^{2}}-2 q \sinh q x \cosh q x \frac{d}{d x}+q^{2} l(l+2) \cosh ^{2} q x+q^{2} k(k-1) \operatorname{csch}^{2} q x\right) \\
& \times \phi_{n, l}^{(k)}(x) \\
= & \left(E_{n, l}^{(k)}-q^{2} v_{0}\right) \phi_{n, l}^{(k)}(x)
\end{aligned}
$$


and $n$ distinguishes between the independent solutions characterized by the same eigenvalue of $L$.

By the changes of variable and of function

$$
\begin{gathered}
e^{q x}=\tan \left[\frac{1}{2}\left(z+\frac{\pi}{2}\right)\right], \quad 0<z<\frac{\pi}{2}, \\
\phi_{n, l}^{(k)}[x(z)]=\sqrt{\cos z} \xi_{n}^{(\kappa, \lambda)}(z),
\end{gathered}
$$

Eq. (3.12) can be mapped onto the (constant-mass) Schrödinger equation for the twoparameter trigonometric Pöschl-Teller potential (also termed Pöschl-Teller I potential) [32]

$$
\left(-\frac{d^{2}}{d z^{2}}+\kappa(\kappa-1) \csc ^{2} z+\lambda(\lambda-1) \sec ^{2} z\right) \xi_{n}^{(\kappa, \lambda)}(z)=\mathcal{E}_{n}^{(\kappa, \lambda)} \xi_{n}^{(\kappa, \lambda)}(z)
$$

where

$$
\kappa=k, \quad \lambda=l+\frac{3}{2}, \quad \mathcal{E}_{n}^{(\kappa, \lambda)}=\frac{1}{q^{2}}\left[E_{n, l}^{(k)}-q^{2} v_{0}+q^{2}\left(k-\frac{1}{2}\right)^{2}\right] .
$$

From the known solutions of the latter [29, 33] in terms of Jacobi polynomials, we obtain for the former

$$
E_{n, l}^{(k)}=q^{2}\left[(2 n+l+2)(2 n+l+2 k+1)+v_{0}\right]
$$

and

$$
\begin{aligned}
\phi_{n, l}^{(k)} & =\mathcal{N}_{n, l}^{(k)}(\tanh q x)^{k}(\operatorname{sech} q x)^{l+2} P_{n}^{\left(k-\frac{1}{2}, l+1\right)}\left(1-2 \tanh ^{2} q x\right), \\
\mathcal{N}_{n, l}^{(k)} & =\left(\frac{2 q\left(2 n+l+k+\frac{3}{2}\right) n ! \Gamma\left(n+l+k+\frac{3}{2}\right)}{(n+l+1) ! \Gamma\left(n+k+\frac{1}{2}\right)}\right)^{1 / 2} .
\end{aligned}
$$

The functions (3.11) with $\phi_{n, l}^{(k)}(x)$ and $\chi_{l}(y)$ given in Eqs. (3.18) and (3.10), respectively, are square integrable over $D$ with the normalization factors as indicated. Since the PDM $M(x)=\operatorname{sech}^{2} q x$ vanishes for $x \rightarrow \infty$, such functions will describe bound states only if condition (2.5) is satisfied in this limit. This is indeed the case because $\cosh q x\left|\phi_{n, l}^{(k)}(x)\right|^{2} \sim$ $\exp [-(2 l+3) q x]$ for $x \rightarrow \infty$.

We conclude that the bound-state spectrum of $H^{(k)}$ is made of an infinite number of energy levels, given by (3.17). Since $E_{n, l}^{(k)}$ only depends on the combination

$$
N=2 n+l,
$$


we can rewrite it as

$$
E_{N}^{(k)} \equiv E_{n, l}^{(k)}=q^{2}\left[(N+2)(N+2 k+1)+v_{0}\right]
$$

The level characterized by $N$ comprises the states specified by $(n, l)=(0, N),(1, N-2), \ldots$, $\left(\frac{N}{2}, 0\right)$ or $\left(\frac{N-1}{2}, 1\right)$ according to whether $N$ is even or odd. Its degeneracy is therefore given by

$$
\operatorname{deg}(N)=\left[\frac{N}{2}\right]+1
$$

where $[N / 2]$ stands for the integer part of $N / 2$.

Before going to the alternative approach based upon the intertwining operators $\eta^{(k)}$ and $\eta^{(k) \dagger}$ in Subsection 3.2, it is worth making a pause for observing that the one-dimensional Hamiltonian $H_{l}^{(k)}$, as defined in (3.12), itself satisfies some intertwining relationships

$$
\mathcal{A}_{l}^{(k)} H_{l}^{(k)}=\left(H_{l+1}^{(k+1)}+2 q^{2} k\right) \mathcal{A}_{l}^{(k)}, \quad \tilde{\mathcal{A}}_{l}^{(k)} H_{l}^{(k)}=\left(H_{l-1}^{(k+1)}+2 q^{2} k\right) \tilde{\mathcal{A}}_{l}^{(k)}
$$

with the first-order differential operators

$$
\begin{gathered}
\mathcal{A}_{l}^{(k)}=\cosh q x \frac{d}{d x}+q(l+2) \sinh q x-q k \operatorname{csch} q x, \\
\tilde{\mathcal{A}}_{l}^{(k)}=\cosh q x \frac{d}{d x}-q l \sinh q x-q k \operatorname{csch} q x .
\end{gathered}
$$

Such a property derives from the existence for $H_{l}^{(k)}$ of a four-way factorization

$$
\begin{aligned}
H_{l}^{(k)} & =\mathcal{A}_{l}^{(k) \dagger} \mathcal{A}_{l}^{(k)}+c_{l}^{(k)}=\mathcal{A}_{l-1}^{(k-1)} \mathcal{A}_{l-1}^{(k-1) \dagger}+c_{l-2}^{(k)} \\
& =\tilde{\mathcal{A}}_{l}^{(k) \dagger} \tilde{\mathcal{A}}_{l}^{(k)}+\tilde{c}_{l}^{(k)}=\tilde{\mathcal{A}}_{l+1}^{(k-1)} \tilde{\mathcal{A}}_{l+1}^{(k-1) \dagger}+\tilde{c}_{l+2}^{(k)} \\
c_{l}^{(k)} & \equiv q^{2}(l+2)(l+2 k+1), \quad \tilde{c}_{l}^{(k)} \equiv q^{2} l(l-2 k+1) .
\end{aligned}
$$

The latter is similar to that occurring for the three-dimensional radial harmonic oscillator [34] and is directly connected with the existence of an so(4) potential algebra for the two-parameter trigonometric Pöschl-Teller potential [33].

In a standard (unbroken) SUSYQM approach to $H_{l}^{(k)}$, one would only consider the pair of operators $\mathcal{A}_{l}^{(k)}$ and $\mathcal{A}_{l}^{(k) \dagger}$. The former indeed annihilates the ground-state wavefunction $\phi_{0, l}^{(k)}$ of $H_{l}^{(k)}$ and changes $\phi_{n, l}^{(k)}$ into $\phi_{n-1, l+1}^{(k+1)}$ for $n=1,2, \ldots$, while the latter generates transitions between $\phi_{n, l+1}^{(k+1)}$ and $\phi_{n+1, l}^{(k)}$ for $n=0,1,2, \ldots$ In contrast, the second pair of operators $\tilde{\mathcal{A}}_{l}^{(k)}$ and $\tilde{\mathcal{A}}_{l}^{(k) \dagger}$ does not change the $n$ value as one goes this time from $\phi_{n, l}^{(k)}$ to $\phi_{n, l-1}^{(k+1)}$ for $n=0,1,2, \ldots$, or vice versa. 
Interpreting these results in terms of the full two-dimensional Hamiltonian $H^{(k)}$ is difficult because one is confronted with the same type of problem as that occurring in the SUSYQM approach to the Coulomb problem [29]. In such a case, the intertwining operators for the radial Hamiltonian change the angular momentum quantum number $l$ by one unit without affecting the angular part $Y_{l, m}(\theta, \varphi)$ of the wavefunction. As a consequence, there has been much controversy on how to apply the SUSYQM results to atomic spectra [35. Here too we may observe that both intertwining operators $\mathcal{A}_{l}^{(k)}, \tilde{\mathcal{A}}_{l}^{(k)}$ and their Hermitian conjugates change by one unit the quantum number $l$, now connected with the eigenvalue of the operator $L$ defined in (3.8), although the corresponding eigenfunction $\chi_{l}(y)$ remains the same.

As we plan to show in Subsection 3.3, the intertwining operators $\eta^{(k)}$ and $\eta^{(k) \dagger}$ offer the advantage of being free from such a problem since they act on $\chi_{l}(y)$ as well as on $\phi_{n, l}^{(k)}(x)$.

\subsection{Intertwining Operator Approach}

As established in Section 2, the operators $H^{(k)}$ and $R^{(k)}$ defined in (3.3) and (3.6), respectively, are simultaneously diagonalizable. Let us denote their simultaneous eigenfunctions by $\Psi_{N, N_{0}}^{(k)}(x, y)$ and the corresponding eigenvalues by $E_{N}^{(k)}$ and $r_{\nu}^{(k)}$, with $N \equiv N_{0}+\nu$. Hence

$$
H^{(k)} \Psi_{N, N_{0}}^{(k)}(x, y)=E_{N}^{(k)} \Psi_{N, N_{0}}^{(k)}(x, y), \quad R^{(k)} \Psi_{N, N_{0}}^{(k)}(x, y)=r_{\nu}^{(k)} \Psi_{N, N_{0}}^{(k)}(x, y),
$$

where $r_{\nu}^{(k)} \geq 0$ since $R^{(k)}$ is a positive-definite operator. We plan to show herebelow that in (3.26),$N$ and $\nu$ run over all nonnegative integers, while $N_{0}$ is restricted to nonnegative even integers.

Our first step consists in constructing the functions $\Psi_{N_{0}, N_{0}}^{(k)}(x, y)$ corresponding to $E_{N_{0}}^{(k)}$ and $r_{0}^{(k)}=0$. Since they belong to the subspace spanned by the zero modes of $\eta^{(k)}$, let us inquire into such zero modes and therefore consider the equation

$$
\eta^{(k)} \omega_{s}^{(k)}(x, y)=0
$$

where $s$ will serve to distinguish between independent solutions. From (3.5), it follows that this first-order partial differential equation is separable. Up to some multiplicative constant, its general solution is given by

$$
\omega_{s}^{(k)}(x, y)=(\tanh q x)^{k}(\operatorname{sech} q x)^{s+1}(\cos q y)^{s},
$$


where $s$ is related to the separation constant. To get functions satisfying the boundary conditions (3.2), we have to restrict $s$ to positive values. It is then easy to check that $\omega_{s}^{(k)}(x, y)$ is normalizable on the domain $D$, defined in (3.1), and satisfies condition (2.5) for $x \rightarrow \infty$.

On applying $H^{(k)}$ on the zero modes (3.28), we obtain after a straightforward calculation

$$
H^{(k)} \omega_{s}^{(k)}(x, y)=q^{2}\left[(s+1)(2 k+s)+v_{0}\right] \omega_{s}^{(k)}(x, y)-q^{2} s(s-1) \omega_{s-2}^{(k)}(x, y) .
$$

Hence, amongst the zero modes $\omega_{s}^{(k)}(x, y)$ with $s>0$, there is only one that is an eigenfunction of $H^{(k)}$, namely $\omega_{1}^{(k)}(x, y)$ :

$$
H^{(k)} \omega_{1}^{(k)}(x, y)=q^{2}\left[2(2 k+1)+v_{0}\right] \omega_{1}^{(k)}(x, y) .
$$

As it can be checked by comparison with (3.20) and with (3.10), (3.11), (3.18), the eigenvalue and eigenfunction of $H^{(k)}$ in (3.30) coincide with the ground-state energy $E_{0}^{(k)}$ and groundstate wavefunction $\psi_{0,0}^{(k)}(x, y)$, respectively. We have therefore found the (unique) solution of (3.26) with $r_{0}^{(k)}=0$ and $N=N_{0}=0$,

$$
\Psi_{0,0}^{(k)}(x, y)=\psi_{0,0}^{(k)}(x, y) \propto \omega_{1}^{(k)}(x, y) .
$$

We can now get the remaining solutions of (3.26) with $r_{0}^{(k)}=0$ and $N=N_{0} \neq 0$ by looking for those linear combinations of $\omega_{s}^{(k)}(x, y), s=1,3, \ldots, N_{0}+1$,

$$
\Psi_{N_{0}, N_{0}}^{(k)}(x, y)=\sum_{s=1}^{N_{0}+1} \frac{1}{2}\left[1-(-1)^{s}\right] a_{s}^{(k)} \omega_{s}^{(k)}(x, y),
$$

that are eigenfunctions of $H^{(k)}$. Note that in (3.32), $N_{0}$ is necessarily restricted to even integers. On using (3.29), we obtain a recursion relation for the coefficients $a_{s}^{(k)}$ together with the eigenvalue of $H^{(k)}$,

$$
E_{N_{0}}^{(k)}=q^{2}\left[\left(N_{0}+2\right)\left(N_{0}+2 k+1\right)+v_{0}\right] .
$$

The solution to the recursion relation reads

$$
a_{s}^{(k)}=(-1)^{\left(N_{0}+1-s\right) / 2} \frac{\left(N_{0}+1\right) ! \Gamma\left(\frac{N_{0}+s}{2}+k+1\right)}{2^{N_{0}+1-s} s !\left(\frac{N_{0}-s+1}{2}\right) ! \Gamma\left(N_{0}+k+\frac{3}{2}\right)} a_{N_{0}+1}^{(k)}, \quad s=1,3, \ldots, N_{0}-1 .
$$

The remaining multiplicative factor $a_{N_{0}+1}^{(k)}$ can in principle be determined through the normalization condition of $\Psi_{N_{0}, N_{0}}^{(k)}(x, y)$ on $D$. However, since the zero modes $\omega_{s}^{(k)}(x, y), s=1$, 
$3,5, \ldots$, form a nonorthogonal set, this condition is not easy to work out. We shall therefore leave the construction of fully normalized functions $\Psi_{N_{0}, N_{0}}^{(k)}(x, y)$ to Subsection 3.3, where they will be expressed in an orthogonal basis.

In a second step, we can now obtain from the functions $\Psi_{N_{0}, N_{0}}^{(k)}(x, y)$ the solutions of (3.26) associated to $\nu=1,2, \ldots$, and correspondingly $N=N_{0}+1, N_{0}+2, \ldots$, by a straightforward application of the intertwining relations (2.6) and (2.15) for the operators $\eta^{(k)}, \eta^{(k) \dagger}, H^{(k)}, H_{1}^{(k)}, R^{(k)}$, and $R_{1}^{(k)}$. On comparing (3.4) and (3.7) with (3.3) and (3.6), respectively, we indeed observe that

$$
\begin{gathered}
H_{1}^{(k)}=H^{(k+1)}+\epsilon^{(k)}, \quad \epsilon^{(k)}=2 q^{2} k, \\
R_{1}^{(k)}=R^{(k+1)}+\rho^{(k)}, \quad \rho^{(k)}=q^{2}(2 k+1) .
\end{gathered}
$$

In other words, $H^{(k)}$ and $R^{(k)}$ are both shape invariant [29]. In view of this property, we directly obtain

$$
\Psi_{N, N_{0}}^{(k)}(x, y)=\overline{\mathcal{N}}_{N, N_{0}}^{(k)} \eta^{(k) \dagger} \eta^{(k+1) \dagger} \cdots \eta^{(k+\nu-1) \dagger} \Psi_{N_{0}, N_{0}}^{(k+\nu)}(x, y), \quad \nu=1,2, \ldots,
$$

where $\overline{\mathcal{N}}_{N, N_{0}}^{(k)}$ is some normalization coefficient and

$$
\begin{gathered}
E_{N}^{(k)}=E_{N_{0}}^{(k+\nu)}+\sum_{i=0}^{\nu-1} \epsilon^{(k+i)}=q^{2}\left[(N+2)(N+2 k+1)+v_{0}\right], \\
r_{\nu}^{(k)}=\sum_{i=0}^{\nu-1} \rho^{(k+i)}=q^{2} \nu(\nu+2 k),
\end{gathered}
$$

for $N=N_{0}+\nu$. Note that the eigenvalues (3.38) of $H^{(k)}$ coincide with those previously found in (3.20) as it should be. However, the supersymmetric approach adopted in this Subsection has the advantage of explaining the dependence of these eigenvalues on a single quantum number $N$, which otherwise would appear to be accidental.

As a final point, let us observe that the normalization coefficient $\overline{\mathcal{N}}_{N, N_{0}}^{(k)}$ in (3.37) can be easily derived from the property

$$
\left\langle\Psi_{N, N_{0}}^{(k)} \mid \Psi_{N, N_{0}}^{(k)}\right\rangle=\left(\frac{\overline{\mathcal{N}}_{N, N_{0}}^{(k)}}{\overline{\mathcal{N}}_{N-1, N_{0}}^{(k+1)}}\right)^{2}\left\langle\Psi_{N-1, N_{0}}^{(k+1)}\left|\eta^{(k)} \eta^{(k) \dagger}\right| \Psi_{N-1, N_{0}}^{(k+1)}\right\rangle
$$

and Eqs. (2.12), (3.26), (3.36), (3.39). The result reads

$$
\overline{\mathcal{N}}_{N, N_{0}}^{(k)}=q^{-\nu}\left(\frac{\Gamma(2 k+\nu)}{\nu ! \Gamma(2 k+2 \nu)}\right)^{1 / 2}, \quad N=N_{0}+\nu .
$$




\subsection{Relation between the Two Approaches}

In Subsections 3.1 and 3.2, we followed two different methods for determining the boundstate spectrum and wavefunctions of $H^{(k)}$. The former was based on the separability and exact solvability of the corresponding Schrödinger equation, while the latter used an intertwining-operator approach and its supersymmetric interpretation. Such methods resulted in two distinct bases for the associated Hilbert space, $\psi_{n, l}^{(k)}(x, y), n, l=0,1,2, \ldots$, and $\Psi_{N, N_{0}}^{(k)}(x, y), N_{0}=0,2,4, \ldots, N=N_{0}, N_{0}+1, N_{0}+2, \ldots$, corresponding to definite eigenvalues of the respective symmetry operator, $L$ or $R^{(k)}$. The aim of this Subsection is to determine the transformation matrix between these two bases.

For such a purpose, let us first consider the action of the intertwining operators $\eta^{(k)}$ and $\eta^{(k) \dagger}$ on the first basis wavefunctions $\psi_{n, l}^{(k)}(x, y)$. As it is clear from the analysis of Subsection 3.2 (see Eq. (3.37)), such operators have the property of decreasing or increasing $N=2 n+l$ by one unit, while changing $k$ into $k+1$ or vice versa. Hence, when acting on some generic function $\psi_{n, l}^{(k)}(x, y), \eta^{(k)}$, for instance, will generate transitions to $\psi_{n-1, l+1}^{(k+1)}(x, y)$ and $\psi_{n, l-1}^{(k+1)}(x, y)$. This is confirmed by an explicit calculation using Eqs. (3.5), (3.10), (3.11), (3.18), and some well-known properties of Jacobi polynomials [36]. The obtained result reads

$$
\begin{aligned}
\eta^{(k)} \psi_{n, l}^{(k)}= & (-1)^{N} q\left[-\sqrt{n\left(n+l+k+\frac{3}{2}\right)} \psi_{n-1, l+1}^{(k+1)}\right. \\
& \left.+\left(1-\delta_{l, 0}\right) \sqrt{\left(n+k+\frac{1}{2}\right)(n+l+1)} \psi_{n, l-1}^{(k+1)}\right] .
\end{aligned}
$$

Similarly, for the Hermitian conjugate operator $\eta^{(k) \dagger}$ we get

$$
\begin{aligned}
\eta^{(k) \dagger} \psi_{n, l}^{(k+1)}= & (-1)^{N+1} q\left[-\left(1-\delta_{l, 0}\right) \sqrt{(n+1)\left(n+l+k+\frac{3}{2}\right)} \psi_{n+1, l-1}^{(k)}\right. \\
& \left.+\sqrt{\left(n+k+\frac{1}{2}\right)(n+l+2)} \psi_{n, l+1}^{(k)}\right] .
\end{aligned}
$$

We are now able to expand the members of the second basis that are zero modes of $\eta^{(k)}$, i.e., $\Psi_{N_{0}, N_{0}}^{(k)}(x, y), N_{0}=0,2,4, \ldots$, into linear combinations of the first basis wavefunctions $\psi_{n, l}^{(k)}(x, y)$ with $2 n+l=N_{0}$,

$$
\Psi_{N_{0}, N_{0}}^{(k)}(x, y)=\sum_{n=0}^{N_{0} / 2} X_{n, N_{0}-2 n}^{(k)} \psi_{n, N_{0}-2 n}^{(k)}(x, y), \quad N_{0}=0,2,4, \ldots,
$$


where $X_{n, N_{0}-2 n}^{(k)}$ are some coefficients to be determined. On applying $\eta^{(k)}$ on both sides of this equation and using the facts that the left-hand side vanishes while Eq. (3.42) allows us to calculate the right-hand one, we indeed obtain a recursion relation for the coefficients $X_{n, N_{0}-2 n}^{(k)}$, whose solution is given by

$$
X_{n, N_{0}-2 n}^{(k)}=\left(\frac{\left(\frac{N_{0}}{2}\right) !\left(\frac{N_{0}}{2}+1\right) ! \Gamma\left(N_{0}-n+k+\frac{3}{2}\right) \Gamma\left(n+k+\frac{1}{2}\right)}{n !\left(N_{0}-n+1\right) ! \Gamma\left(\frac{N_{0}+3}{2}+k\right) \Gamma\left(\frac{N_{0}+1}{2}+k\right)}\right)^{1 / 2} X_{N_{0} / 2,0}^{(k)} .
$$

As shown in the Appendix (see Eq. (A.3)), the remaining multiplicative factor $X_{N_{0} / 2,0}^{(k)}$ can be computed from the normalization condition of $\Psi_{N_{0}, N_{0}}^{(k)}(x, y)$. The final result for $X_{n, N_{0}-2 n}^{(k)}$ can then be written as

$$
X_{n, N_{0}-2 n}^{(k)}=\left(\frac{\left(N_{0}+1\right) ! \Gamma(k+1) \Gamma\left(n+k+\frac{1}{2}\right) \Gamma\left(N_{0}-n+k+\frac{3}{2}\right)}{2^{N_{0}} n !\left(N_{0}-n+1\right) ! \Gamma\left(\frac{N_{0}}{2}+k+1\right) \Gamma\left(k+\frac{1}{2}\right) \Gamma\left(\frac{N_{0}+3}{2}+k\right)}\right)^{1 / 2} .
$$

For the first few $N_{0}$ values, Eq. (3.44) reads

$$
\begin{gathered}
\Psi_{0,0}^{(k)}=\psi_{0,0}^{(k)} \\
\Psi_{2,2}^{(k)}=\frac{1}{2 \sqrt{k+1}}\left(\sqrt{k+\frac{5}{2}} \psi_{0,2}^{(k)}+\sqrt{3\left(k+\frac{1}{2}\right)} \psi_{1,0}^{(k)}\right) \\
\Psi_{4,4}^{(k)}=\frac{1}{4 \sqrt{(k+1)(k+2)}}\left(\sqrt{\left(k+\frac{7}{2}\right)\left(k+\frac{9}{2}\right)} \psi_{0,4}^{(k)}+\sqrt{5\left(k+\frac{1}{2}\right)\left(k+\frac{7}{2}\right)} \psi_{1,2}^{(k)}\right. \\
\left.+\sqrt{10\left(k+\frac{1}{2}\right)\left(k+\frac{3}{2}\right)} \psi_{2,0}^{(k)}\right) .
\end{gathered}
$$

Note that Eq. (3.47) coincides with (3.31) as it shoud be.

To be able to extend expansion (3.44) to all members $\Psi_{N, N_{0}}^{(k)}(x, y)$ of the second basis, defined in (3.37), we have first to iterate Eq. (3.43) to determine the action of $\nu$ operators of type $\eta^{(k) \dagger}$ on the first basis wavefunctions:

$$
\eta^{(k) \dagger} \eta^{(k+1) \dagger} \cdots \eta^{(k+\nu-1) \dagger} \psi_{n, l}^{(k+\nu)}=\sum_{\mu=0}^{\nu} Y_{n, l ; n+\mu, l+\nu-2 \mu}^{(k)} \psi_{n+\mu, l+\nu-2 \mu}^{(k)} .
$$

Here the coefficients are given by

$$
\begin{aligned}
& Y_{n, l ; n+\mu, l+\nu-2 \mu}^{(k)}=(-1)^{\nu\left(N+\frac{\nu+1}{2}\right)+\mu} q^{\nu} x_{\mu}(l, \nu) \\
& \times\left(\frac{(n+\mu) !(n+l+\nu-\mu+1) ! \Gamma\left(n+\nu+k+\frac{1}{2}\right) \Gamma\left(n+l+\nu+k+\frac{3}{2}\right)}{n !(n+l+1) ! \Gamma\left(n+\mu+k+\frac{1}{2}\right) \Gamma\left(n+l+\nu-\mu+k+\frac{3}{2}\right)}\right)^{1 / 2}
\end{aligned}
$$


where $x_{\mu}(l, \nu)$ is a combinatorial factor giving the number of ways one can go from $l$ to $l+\nu-2 \mu$ in $\nu$ steps so that in each step $l$ increases or decreases by one unit without reaching negative values. This means in particular that

$$
x_{\mu}(l, \nu)=0 \quad \text { if } \mu>\left[\frac{l+\nu}{2}\right] .
$$

Hence the summation on the right-hand side of (3.50) actually runs from 0 to $\min \left(\nu,\left[\frac{l+\nu}{2}\right]\right)$.

For $\nu=1$, Eq. (3.50) reduces to Eq. (3.43) since in such a case

$$
x_{0}(l, 1)=1, \quad x_{1}(l, 1)=1-\delta_{l, 0} .
$$

For higher $\nu$ values, it can be easily proved by induction over $\nu$ on taking the relations

$$
\begin{aligned}
& x_{0}(l, \nu)=x_{0}(l, \nu-1), \\
& x_{\mu}(l, \nu)=\left(1-\delta_{\mu,(l+\nu+1) / 2}\right) x_{\mu-1}(l, \nu-1)+x_{\mu}(l, \nu-1), \quad \mu=1,2, \ldots, \nu-1, \\
& x_{\nu}(l, \nu)=\left(1-\delta_{l, \nu-1}\right) x_{\nu-1}(l, \nu-1)
\end{aligned}
$$

into account. Some examples of explicit solutions to this set of recursion relations are provided in the Appendix.

Finally, it remains to combine Eqs. (3.37), (3.44), and (3.50) to obtain the searched for expansion

$$
\Psi_{N, N_{0}}^{(k)}=\sum_{n=0}^{[N / 2]} Z_{N_{0} ; n, N-2 n}^{(k)} \psi_{n, N-2 n}^{(k)},
$$

where

$$
Z_{N_{0} ; n, N-2 n}^{(k)}=\overline{\mathcal{N}}_{N, N_{0}}^{(k)} \sum_{n^{\prime}=n_{\min }^{\prime}}^{n_{\max }^{\prime}} X_{n^{\prime}, N_{0}-2 n^{\prime}}^{(k+\nu)} Y_{n^{\prime}, N_{0}-2 n^{\prime} ; n, N-2 n}^{(k)} .
$$

Here $n_{\min }^{\prime} \equiv \max \left(0, n+N_{0}-N\right), n_{\max }^{\prime} \equiv \min \left(\frac{N_{0}}{2}, n\right)$ and the three factors on the right-hand side are given by Eqs. (3.41), (3.46) and (3.51), respectively.

For those wavefunctions of the second basis $\Psi_{N, 0}^{(k)}(x, y)$ that can be reached from the ground state (3.47) by applying $N$ operators of type $\eta^{(k) \dagger}$, Eq. (3.56) can be written explicitly as

$$
\begin{aligned}
& Z_{0 ; n, N-2 n}^{(k)}=(-1)^{\frac{1}{2} N(N+3)+n}(N-2 n+1) \\
& \times\left(\frac{N ! \Gamma\left(\frac{N}{2}+k\right) \Gamma\left(\frac{N+1}{2}+k\right) \Gamma\left(N+k+\frac{3}{2}\right)}{2^{N} n !(N-n+1) ! \Gamma(N+k) \Gamma\left(n+k+\frac{1}{2}\right) \Gamma\left(N-n+k+\frac{3}{2}\right)}\right)^{1 / 2}
\end{aligned}
$$


on using Eq. (A.8) for the combinatorial factor $x_{\mu}(0, \nu)$. So, for instance,

$$
\begin{gathered}
\Psi_{1,0}^{(k)}=\psi_{0,1}^{(k)} \\
\Psi_{2,0}^{(k)}=\frac{1}{2 \sqrt{k+1}}\left(-\sqrt{3\left(k+\frac{1}{2}\right)} \psi_{0,2}^{(k)}+\sqrt{k+\frac{5}{2}} \psi_{1,0}^{(k)}\right) \\
\Psi_{3,0}^{(k)}=\frac{1}{\sqrt{2(k+2)}}\left(-\sqrt{k+\frac{1}{2}} \psi_{0,3}^{(k)}+\sqrt{k+\frac{7}{2}} \psi_{1,1}^{(k)}\right) \\
\Psi_{4,0}^{(k)}=\frac{1}{4 \sqrt{(k+2)(k+3)}}\left(\sqrt{5\left(k+\frac{1}{2}\right)\left(k+\frac{3}{2}\right)} \psi_{0,4}^{(k)}-3 \sqrt{\left(k+\frac{3}{2}\right)\left(k+\frac{9}{2}\right)} \psi_{1,2}^{(k)}\right. \\
\left.+\sqrt{2\left(k+\frac{7}{2}\right)\left(k+\frac{9}{2}\right)} \psi_{2,0}^{(k)}\right)
\end{gathered}
$$

As it can be checked, the functions (3.48) and (3.59), as well as (3.49) and (3.61), are orthogonal.

\section{FINAL REMARKS}

In this paper, we have developed a general framework for analyzing $d$-dimensional PDM Hamiltonian pairs $\left(H, H_{1}\right)$ admitting a first-order intertwining operator $\eta$. We have established that for $d \geq 2$, there always exists another pair of intertwined second-order partial differential operators $\left(R, R_{1}\right)$ associated with the same operator $\eta$ and such that $R$ (resp. $R_{1}$ ) commutes with $H$ (resp. $H_{1}$ ). We have shown that in a SUSYQM context based on an $\operatorname{sl}(1 / 1)$ superalgebra, $R$ and $R_{1}$ can be interpreted as SUSY partners, while $H$ and $H_{1}$ are related to the Casimir operator of a larger gl(1/1) superalgebra. Furthermore, we have derived a system of partial differential equations to be satisfied by the mass, the functions appearing in the definition of $\eta$ and the effective potentials contained in $H$ and $H_{1}$.

Considering this system in more detail in the two-dimensional case for a PDM depending on a single variable, we have proved that the mass may be of a hyperbolic, trigonometric or rational nature. We have then chosen the class of hyperbolic PDM's and, more specifically, a sech ${ }^{2}$-mass, which has proved very useful in the context of exactly-solvable one-dimensional problems [19, 21]. Under this assumption, we have obtained the general solution to the system of partial differential equations when some integration constant $(G$ in (2.36) ) vanishes. 
From such a solution, we have built a physically-relevant model depicting the motion of a particle in a semi-infinite layer. The corresponding Hamiltonian $H$ has two noncommuting integrals of motion, $L$ and $R$. Diagonalizing $H$ and $L$ simultaneously amounts to separating the Schrödinger equation into two exactly-solvable differential equations. On proceeding along these lines, we have obtained a bound-state spectrum made of an infinite number of levels exhibiting some finite degeneracies. Choosing next to simultanously diagonalize the shape-invariant operators $H$ and $R$, we have taken advantage of poweful SUSYQM techniques to obtain their respective spectrum and eigenfunctions in a straightforward way. The latter approach has had two interesting outcomes. First, it has shed some light on the origin of the 'accidental' degeneracies observed in the Hamiltonian spectrum derived in the former approach. Second, it has provided us with a partnership between the full twodimensional Hamiltonians $H$ and $H_{1}$, free from the interpretation problems that plague the more traditional description based on one-dimensional intertwining operators [35].

Had we chosen the class (2.31) of trigonometric PDM's instead of that of hyperbolic ones, a solution to the system of partial differential equations similar to that obtained in Section 2 would have emerged. The counterpart of the model considered in Section 3, now depicting a particle moving in a rectangular box, however turns out to be devoid of bound states. Constructing some physically-relevant model in such a case therefore remains under study.

Finally, in view of an equivalence between Schrödinger equations involving a PDM and those in a curved space [7, selecting the class (2.30) of rational PDM's and, more specifically, a $1 / x^{2}$-mass would have led to some results directly connected with those recently obtained for intertwined Hamiltonians in Poincaré half plane $\left(A d S_{2}\right)$ [37].

From a physical viewpoint, our model might find applications in the study of quantum wires with an abrupt termination in an environment that can be modelled by a dependence of the carrier effective mass on the position. The presence of bound states may be compared to that observed in a quantum channel whenever the uniformity is broken, for instance by a quantum dot or a bend (see, e.g., 38, and references quoted therein).

Some interesting open problems for future work are the extensions of the present study to higher-dimensional models and to second-order intertwining operators (for a recent review on the latter see, e.g., 39]). 


\section{APPENDIX}

In this Appendix, we prove some results used in the calculation of the transformation matrix between the two bases $\left\{\psi_{n, l}^{(k)}\right\}$ and $\left\{\Psi_{N, N_{0}}^{(k)}\right\}$ in Subsection 3.3.

Let us first consider the computation of the coefficient $X_{N_{0} / 2,0}^{(k)}$ in expansion (3.44). On using Eq. (3.45), the normalization condition $\sum_{n=0}^{N_{0} / 2}\left(X_{n, N_{0}-2 n}^{(k)}\right)^{2}=1$ of $\Psi_{N_{0}, N_{0}}^{(k)}(x, y)$ can be written in terms of

$$
S_{N_{0}}^{(k)}=\sum_{n=0}^{N_{0} / 2} \frac{\Gamma\left(N_{0}-n+k+\frac{3}{2}\right) \Gamma\left(n+k+\frac{1}{2}\right)}{n !\left(N_{0}-n+1\right) !}=\frac{1}{2} \sum_{n=0}^{N_{0}+1} \frac{\Gamma\left(N_{0}-n+k+\frac{3}{2}\right) \Gamma\left(n+k+\frac{1}{2}\right)}{n !\left(N_{0}-n+1\right) !} .
$$

The identity $(1+x)^{-\left(k+\frac{1}{2}\right)}(1+x)^{-\left(k+\frac{1}{2}\right)}=(1+x)^{-(2 k+1)}$, combined with the binomial theorem, leads to the closed form

$$
S_{N_{0}}^{(k)}=\frac{\Gamma\left(N_{0}+2 k+2\right) \Gamma^{2}\left(k+\frac{1}{2}\right)}{2\left(N_{0}+1\right) ! \Gamma(2 k+1)} .
$$

From this result and some elementary properties of the gamma function [36], we obtain

$$
X_{N_{0} / 2,0}^{(k)}=\left(\frac{\left(N_{0}+1\right) ! \Gamma\left(\frac{N_{0}+1}{2}+k\right) \Gamma(k+1)}{2^{N_{0}}\left(\frac{N_{0}}{2}\right) !\left(\frac{N_{0}}{2}+1\right) ! \Gamma\left(k+\frac{1}{2}\right) \Gamma\left(\frac{N_{0}}{2}+k+1\right)}\right)^{1 / 2} .
$$

Let us next provide some solutions to the set (3.54) of recursion relations for the combinatorial factor $x_{\mu}(l, \nu)$ with the starting values given in (3.53). The simplest case corresponds to $l \geq \nu$, because in none of the $\nu$ steps from $l$ to $l+\nu-2 \mu$ can a negative $l$ value be reached. From (3.52), it follows that $x_{\mu}(l, \nu)$ is nonvanishing for $\mu=0,1, \ldots, \nu$. Furthermore, Eq. (3.54) reduces to

$$
\begin{aligned}
x_{0}(l, \nu) & =x_{0}(l, \nu-1) \\
x_{\mu}(l, \nu) & =x_{\mu-1}(l, \nu-1)+x_{\mu}(l, \nu-1), \quad \mu=1,2, \ldots, \nu-1, \\
x_{\nu}(l, \nu) & =x_{\nu-1}(l, \nu-1)
\end{aligned}
$$

so that $x_{\mu}(l, \nu)$ is simply given by a binomial coefficient

$$
x_{\mu}(l, \nu)=\left(\begin{array}{c}
\nu \\
\mu
\end{array}\right), \quad \mu=0,1, \ldots, \nu, \quad \text { if } l \geq \nu .
$$


In contrast, the value $l=0$ maximizes the number of possible encounters with negative $l$ values during the $\nu$ steps. The nonvanishing values of $x_{\mu}(l, \nu)$ now correspond to $\mu=0$, $1, \ldots,\left[\frac{\nu}{2}\right]$ and satisfy the relations

$$
\begin{aligned}
x_{0}(0, \nu) & =x_{0}(0, \nu-1), \\
x_{\mu}(0, \nu) & =x_{\mu-1}(0, \nu-1)+x_{\mu}(0, \nu-1), \quad \mu=1,2, \ldots, \frac{\nu}{2}-1, \\
x_{\frac{\nu}{2}}(0, \nu) & =x_{\frac{\nu}{2}-1}(0, \nu-1),
\end{aligned}
$$

or

$$
\begin{aligned}
& x_{0}(0, \nu)=x_{0}(0, \nu-1), \\
& x_{\mu}(0, \nu)=x_{\mu-1}(0, \nu-1)+x_{\mu}(0, \nu-1), \quad \mu=1,2, \ldots, \frac{\nu-1}{2},
\end{aligned}
$$

for even and odd values of $\nu$, respectively. In both cases, the solution reads

$$
x_{\mu}(0, \nu)=\left\{\begin{array}{ll}
\frac{\nu !(\nu-2 \mu+1)}{\mu !(\nu-\mu+1) !} & \mu=0,1, \ldots,\left[\frac{\nu}{2}\right] \\
0 & \mu=\left[\frac{\nu}{2}\right]+1,\left[\frac{\nu}{2}\right]+2, \ldots, \nu .
\end{array} .\right.
$$

\section{ACKNOWLEDGMENT}

The author would like to thank V. M. Tkachuk for an interesting discussion. She is a Research Director of the National Fund for Scientific Research (FNRS), Belgium. 


\section{References}

[1] P. Ring and P. Schuck, "The Nuclear Many Body Problem," Springer, New York, 1980.

[2] F. Arias de Saavedra, J. Boronat, A. Polls, and A. Fabrocini, Phys. Rev. B 50 (1994), 4248.

[3] M. Barranco, M. Pi, S. M. Gatica, E. S. Hernández, and J. Navarro, Phys. Rev. B 56 (1997), 8997.

[4] A. Puente, Ll. Serra, and M. Casas, Z. Phys. D 31 (1994), 283.

[5] G. Bastard, "Wave Mechanics Applied to Semiconductor Heterostructures," Editions de Physique, Les Ulis, 1988.

[6] L. Serra and E. Lipparini, Europhys. Lett. 40 (1997), 667.

[7] C. Quesne and V. M. Tkachuk, J. Phys. A 37 (2004), 4267.

[8] C. M. Bender and S. Boettcher, Phys. Rev. Lett. 80 (1998), 5243; C. M. Bender, D. C. Brody, and H. F. Jones, Phys. Rev. Lett. 89 (2002), 270401; C. M. Bender, D. C. Brody, and H. F. Jones, Phys. Rev. Lett. 92 (2004), 119902(E).

[9] A. Mostafazadeh, J. Math. Phys. 43 (2002), 205; A. Mostafazadeh, J. Phys. A 36 (2003), 7081.

[10] H. F. Jones, J. Phys. A 38 (2005), 1741; A. Mostafazadeh, J. Phys. A 38 (2005), 6557.

[11] L. Dekar, L. Chetouani, and T. F. Hammann, J. Math. Phys. 39 (1998), 2551; L. Dekar, L. Chetouani, and T. F. Hammann, Phys. Rev. A 59 (1999), 107.

[12] V. Milanović and Z. Ikonić, J. Phys. A 32 (1999), 7001.

[13] A. R. Plastino, A. Rigo, M. Casas, F. Garcias, and A. Plastino, Phys. Rev. A 60 (1999), 4318; A. R. Plastino, A. Puente, M. Casas, F. Garcias, and A. Plastino, Rev. Mex. Fis. 46 (2000), 78.

[14] A. de Souza Dutra and C. A. S. Almeida, Phys. Lett. A 275 (2000), 25; A. de Souza Dutra, M. Hott, and C. A. S. Almeida, Europhys. Lett. 62 (2003), 8. 
[15] B. Roy and P. Roy, J. Phys. A 35 (2002), 3961; B. Roy and P. Roy, Phys. Lett. A 340 (2005), 70 .

[16] R. Koç, M. Koca, and E. Körcük, J. Phys. A 35 (2002), L527; R. Koç and M. Koca, J. Phys. A 36 (2003), 8105; R. Koç and H. Tütüncüler, Ann. Phys. (Leipzig) 12 (2003), 684.

[17] A. D. Alhaidari, Phys. Rev. A 66 (2002), 042116.

[18] B. Gönül, B. Gönül, D. Tutcu, and O. Özer, Mod. Phys. Lett. A 17 (2002), 2057; B. Gönül, O. Özer, B. Gönül, and F. Üzgün, Mod. Phys. Lett. A 17 (2002), 2453; B. Gönül and M. Koçak, Remarks on exact solvability of quantum systems with spatially varying effective mass, preprint, quant-ph/0412161.

[19] B. Bagchi, P. Gorain, C. Quesne, and R. Roychoudhury, Mod. Phys. Lett. A 19 (2004), 2765; B. Bagchi, P. Gorain, C. Quesne, and R. Roychoudhury, Czech. J. Phys. 54 (2004), 1019.

[20] B. Bagchi, A. Banerjee, C. Quesne, and V. M. Tkachuk, J. Phys. A 38 (2005), 2929.

[21] B. Bagchi, P. Gorain, C. Quesne, and R. Roychoudhury, New approach to (quasi-)exactly solvable Schrödinger equations with a position-dependent effective mass, preprint, quant-ph/0505171, Europhys. Lett. (in press).

[22] J.Yu, S.-H. Dong, and G.-H. Sun, Phys. Lett. A 322 (2004), 290; J.Yu and S.-H. Dong, Phys. Lett. A 325 (2004), 194.

[23] G. Chen and Z. Chen, Phys. Lett. A 331 (2004), 312.

[24] S.-H. Dong and M. Lozada-Cassou, Phys. Lett. A 337 (2005), 313.

[25] A. D. Alhaidari, Phys. Lett. A 322 (2004), 72.

[26] I. O. Vakarchuk, J. Phys. A 38 (2005), 4727.

[27] A. Bhattarcharjie and E. C. G. Sudarshan, Nuovo Cimento 25 (1962), 864; G. A. Natanzon, Theor. Math. Phys. 38 (1979), 146; G. Lévai, J. Phys. A 22 (1989), 689. 
[28] Y. Alhassid, F. Gürsey, and F. Iachello, Ann. Phys. (N.Y.) 167 (1986), 181; J. Wu and Y. Alhassid, J. Math. Phys. 31 (1990), 557; M. J. Englefield and C. Quesne, J. Phys. A 24 (1991), 3557; G. Lévai, J. Phys. A 27 (1994), 3809.

[29] F. Cooper, A. Khare, and U. Sukhatme, Phys. Rep. 251 (1995), 267; B. Bagchi, "Supersymmetry in Quantum and Classical Mechanics," Chapman and Hall / CRC, Boca Raton, Fl., 2000.

[30] O. von Roos, Phys. Rev. B 27 (1983), 7547.

[31] M. Scheunert, "The Theory of Lie Superalgebras," Springer-Verlag, Berlin, 1979.

[32] G. Pöschl and E. Teller, Z. Physik 83 (1933), 143.

[33] A. O. Barut, A. Inomata, and R. Wilson, J. Phys. A 20 (1987), 4075; C. Quesne, J. Phys. A 21 (1988), 4487.

[34] D. J. Fernández, J. Negro, and M. A. del Olmo, Ann. Phys. (N.Y.) 252 (1996), 386.

[35] V. A. Kostelecký and M. M. Nieto, Phys. Rev. Lett. 53 (1984), 2285; A. R. P. Rau, Phys. Rev. Lett. 56 (1986), 95; V. A. Kostelecký and M. M. Nieto, Phys. Rev. Lett. 56 (1986), 96; R. W. Haymaker and A. R. P. Rau, Am. J. Phys. 54 (1986), 928.

[36] A. Erdélyi, W. Magnus, F. Oberhettinger, and F. G. Tricomi, "Higher Transcendental Functions," McGraw-Hill, New York, 1953.

[37] K. A. Samani and M. Zarei, Ann. Phys. (N.Y.) 316 (2005), 466.

[38] O. Olendski and L. Mikhailovska, Phys. Rev. B 66 (2002), 035331; V. Gudmundsson, C.-S. Tang, and A. Manolescu, Phys. Rev. B 72 (2005), 153306.

[39] M. V. Ioffe, J. Phys. A 37 (2004), 10363. 